\title{
Reissner Plates with Plastic Behavior: Probability of Failure
}

\author{
Ernesto Pineda-León, ${ }^{1}$ Alejandro Rodríguez-Castellanos $\mathbb{D}^{2,3}$ Dante Tolentino, ${ }^{1}$ \\ José Manuel Rosales-Juárez, ${ }^{3}$ Ivan Felix-González, ${ }^{2}$ and Supriyono ${ }^{4}$ \\ ${ }^{1}$ Escuela Superior de Ingeniería y Arquitectura, SEPI-ESIA, Instituto Politécnico Nacional, \\ Unidad Profesional Adolfo López Mateos, s/n, Ciudad de México, Mexico \\ ${ }^{2}$ Instituto Mexicano del Petróleo, Eje Central Lázaro Cárdenas 152, 07730 Gustavo A. Madero, Ciudad de México, Mexico \\ ${ }^{3}$ Escuela Superior de Ingeniería Mecánica y Eléctrica, SEPI-ESIME, Instituto Politécnico Nacional, \\ Unidad Profesional Adolfo López Mateos, s/n, Ciudad de México, Mexico \\ ${ }^{4}$ Department of Mechanical Engineering, Universitas Muhammadiyah Surakarta, Surakarta, Indonesia \\ Correspondence should be addressed to Alejandro Rodríguez-Castellanos; arcastel@imp.mx
}

Received 9 February 2018; Revised 11 April 2018; Accepted 16 April 2018; Published 24 May 2018

Academic Editor: Anna M. Gil-Lafuente

Copyright (C) 2018 Ernesto Pineda-León et al. This is an open access article distributed under the Creative Commons Attribution License, which permits unrestricted use, distribution, and reproduction in any medium, provided the original work is properly cited.

\begin{abstract}
The current paper shows the application of the boundary element method for the analysis of plates under shear stress causing plasticity. In this case, the shear deformation of a plate is considered by means of Reissner's theory. The probability of failure of a Reissner's plate due to a proposed index plastic behavior $I_{\mathrm{PB}}$ is calculated taking into account the uncertainty in mechanical and geometrical properties. The problem is developed in three dimensions. The classic plasticity's theory is applied and a formulation for initial stresses that lead to the boundary integral equations due to plasticity is also used. For the plasticity calculation, the von Misses criterion is used. To solve the nonlinear equations, an incremental method is employed. The results show a relatively small failure probability $\left(P_{F}\right)$ for the ranges of loads between $0.6<\widehat{W}<1.0$. However, for values between $1.0<\widehat{W}<2.5$, the probability of failure increases significantly. Consequently, for $\widehat{W} \geq 2.5$, the plate failure is imminent. The results are compared to those that were found in the literature and the agreement is good.
\end{abstract}

\section{Introduction}

All the materials have an elastic limit state; when the load is above this limit state, the material gets into the plastic deformation. This plastic strain depends not only on the final state of stresses but also on the load history. The plastic strain is nonlinear and is irreversible; hence, the physical relations that define the process are more complicated than those that define the elastic behavior.

Plates are commonly used in civil and mechanical engineering. They are used to fabricate vehicles, airplanes, equipment, slabs, and so forth. The main references with information about the elastic theory with strain due to shear on plates are due to Reissner [1], Mindlin [2], and Timoshenko and Goodier [3]. On the other hand, plasticity theory has been reported by Mendelson [4], Hill [5], and Prager [6].
Additionally, the study of plates has been diversified and more realistic conditions have been studied for its analysis and design. Thermal effects, geometric nonlinearity, explosive loads, plasticity, creep, and probabilistic behavior among others are the kind of nonlinear problems that have been considered for plate analysis.

Recently, several formulations based on ReissnerMindlin plate theory have been developed for the study of foundation plates considering thermal effects [7]. These authors also reported analytical solutions for plates subjected to bending and free vibration. Additional studies on the behavior of foundation plates based on the Reissner-Mindlin theory can be found in [8], Wen and Aliabadi [9], and Jankovski and Skarzauskas [10]. In this last reference, the boundary element method was formulated in the Laplace domain to solve the problem. Also, Nosier and Fallah [11] studied the effect of mechanical and thermal loads on the 


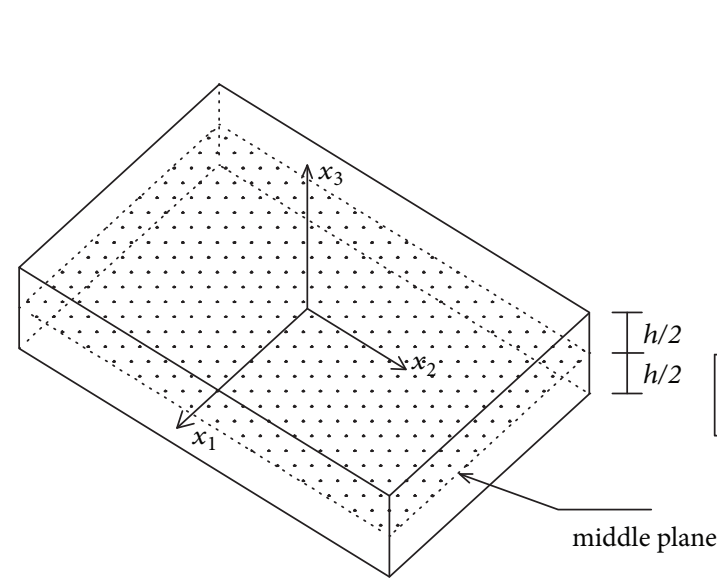

(a)

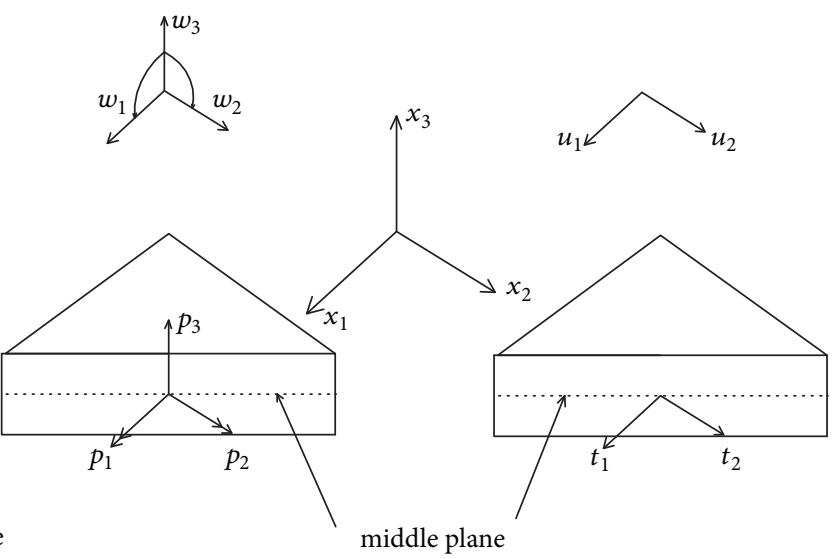

(b)

(c)

Figure 1: (a) Definition of the reference system. (b) Displacements and tractions due to bending and shear. (c) Displacements and tractions in the plane.

axisymmetric and asymmetric behavior of circular plates, where material properties, boundary conditions, plate thickness, and other factors were studied and discussed.

On the other hand, Reissner-Mindlin theory has been applied to the study of nonlinear problems of large deformations on plates. With this idea, Xiao-Yan as well as Mao-kuang and Xiuxi [13] developed an interesting application of the plate boundary element method with geometric nonlinearity in isotropic plates, including the effects of shear deformation. Moreover, recently, Benatta et al. [14] studied plates subjected to pressure loads, where geometric nonlinearity was incorporated into the strain-displacement equations based on the Von-Karman theory. For this case, the results suggest that the precision obtained by this formulation is comparable with high-order theories.

Moreover, Reissner-Mindlin theory has been successfully applied to problems dealing with cracks, composites, and effects of concentrated and uniform loads on plates [15-17], including the study of oceanic plates [18]. In the latter case, the authors numerically solved the flexion equations associated with the Reissner-Mindlin theory and the application was focused on the study of the bending of the Nazca oceanic plate. This fact reveals the power of this theory and how it can be applied to highly complex cases.

Also, there are different factors that modify the response of a structural element; such factors are associated with its mechanical and geometrical properties; that is, there is a probability that the manufacturer will provide a structural element with both geometric and mechanical characteristics different from those specified, which generates uncertainty, since the structural element is designed based on manufacturer's specifications. The loads to which the structural element will be subjected are variable in magnitude, occurrence, direction, and so on. This leads us to consider as stochastic variables all the parameters that intervene in the response of the structural element.

The application of BEM to probabilistic analysis has been successfully applied to solve elastostatic problems [19-22], crack problems [23, 24], and elastic-linear problems [25-27]. Moreover, the BEM method was used for solving optimization problems considering the cost as a parameter of design [28]. Unfortunately, none of the mentioned approaches using BEM have been used to obtain the probability of failure based on a plastic behavior index that considers the relationship between demand and capacity of the structural element. In the present work, the BEM method is used in Reissner plates with plastic behavior and the probability of failure is obtained considering the mechanical and geometrical uncertainties.

In Section 2, the boundary element method for Reissner plates is formulated. The validation of the formulation is included in Section 3. In Section 4, a failure probability approach is given to the analysis of Reissner plates and finally Section 5 includes an application example.

\section{Formulation of the Plastic Problem}

Our formulation is based on Supriyono and Aliabadi [29] and is closely related to Dirgantara and Aliabadi [30]. Furthermore, our research intends to extend the formulation of Supriyono and Aliabadi [29] including the probability of failure of Reissner plates with plastic behavior.

Assuming the reference system of Figure 1(a) and the definition of bending and shear displacements as $w_{i}$ (where $i=1,2,3)$, displacements in the plane as $u_{\alpha}(\alpha=1,2)$ and their associated tractions can be seen in Figures 1(b) and 1(c); for this case, the strain-displacement relations can be written as

$$
\begin{aligned}
\gamma_{\alpha 3} & =\psi_{\alpha}=w_{\alpha}+w_{3, \alpha} \\
\kappa_{\alpha \beta} & =2 \chi_{\alpha \beta}=w_{\alpha, \beta}+w_{\beta, \alpha}, \\
\varepsilon_{\alpha \beta}^{\text {lineal }} & =\frac{1}{2}\left(u_{\alpha, \beta}+u_{\beta, \alpha}\right),
\end{aligned}
$$

where $\gamma_{\alpha 3}$ is transverse shear strain; $\kappa_{\alpha \beta}$ is bending deformation; and $\varepsilon_{\alpha \beta}^{\text {lineal }}$ is strain in the middle plane of the element. 


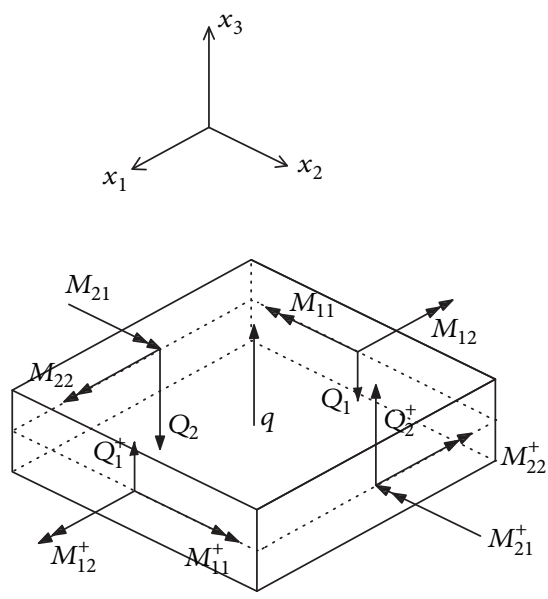

(a)

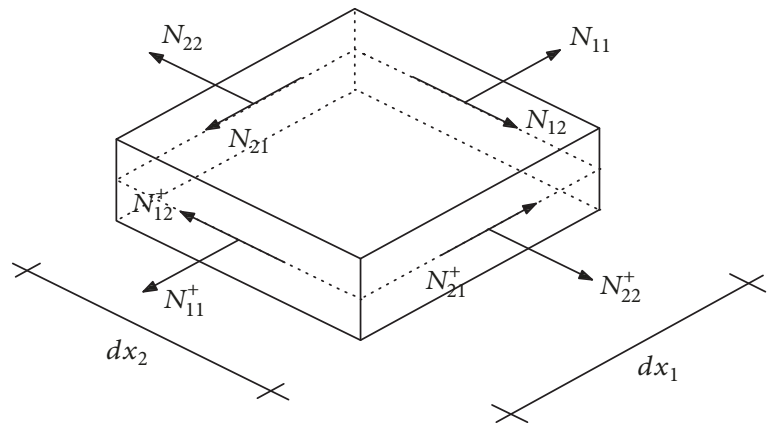

(b)

FIGURE 2: Load's equilibrium on an element.

On one hand, if we consider a differential element of a plate with dimensions $d x_{1} \cdot d x_{2} \cdot h$ subject to a uniform load $q$ (see Figure 2), the equilibrium equations for moments and forces, ignoring higher-order terms, can be expressed as

$$
\begin{array}{r}
M_{\alpha \beta, \beta}-Q_{\alpha}=0 ; \\
Q_{\alpha, \alpha}+q=0 ; \\
N_{\alpha \beta, \beta}^{\text {lineal }}=0,
\end{array}
$$

where $M_{\alpha \beta}$ results from bending stresses, $Q_{\alpha}$ results from shear stresses, and $N_{\alpha \beta}^{\text {lineal }}$ results from membrane stresses.

The notation used in Figure 2 means that $(\ldots)_{12}^{+}$is used to denote $(\ldots)_{12}+\left(\partial(\ldots)_{12} / \partial x_{1}\right) d x_{1}$; the notation $(\ldots)_{21}^{+}$ represents $(\ldots)_{21}+\left(\partial(\ldots)_{21} / \partial x_{2}\right) d x_{2}$.

On the other hand, Reissner [1] obtained the stress-strain relationships for flexural plates, which are

$$
\begin{aligned}
M_{\alpha \beta} & =D \frac{1-\nu}{2}\left(2 \chi_{\alpha \beta}+\frac{2 \nu}{1-\nu} \chi_{\gamma \gamma} \delta_{\alpha \beta}\right) \\
Q_{\alpha} & =C \psi_{\alpha}
\end{aligned}
$$

Based on Hooke's law for plane stresses, the resulting stressstrain relationships for membrane behavior can be expressed as follows:

$$
N_{\alpha \beta}^{\text {lineal }}=B \frac{1-\nu}{2}\left(2 \varepsilon_{\alpha \beta}+\frac{2 \nu}{1-\nu} \varepsilon_{\gamma \gamma} \delta_{\alpha \beta}\right)
$$

In terms of displacements, (7)-(9) are written as

$$
\begin{aligned}
M_{\alpha \beta} & =D \frac{1-v}{2}\left(w_{\alpha, \beta}+w_{\beta, \alpha}+\frac{2 v}{1-\nu} w_{\gamma, \gamma} \delta_{\alpha \beta}\right) \\
Q_{\alpha} & =C\left(w_{\alpha}+w_{3, \alpha}\right) \\
N_{\alpha \beta}^{\text {lineal }} & =B \frac{1-v}{2}\left(u_{\alpha, \beta}+u_{\beta, \alpha}+\frac{2 v}{1-\nu} u_{\gamma, \gamma} \delta_{\alpha \beta}\right),
\end{aligned}
$$

where $B=E h /\left(1-v^{2}\right)=$ tension stiffness, $D=E h^{3} / 12(1-$ $\left.v^{2}\right)=$ flexural stiffness, and $C=5 E h^{3} / 12\left(1-v^{2}\right)=$ shear stiffness. The Kronecker delta is represented by $\delta_{\alpha \beta}$.
2.1. Integral Boundary Formulation for Reissner Plates. According to the equations of equilibrium (4)-(6), it is possible to express the following integral equation:

$$
\begin{array}{r}
\int_{\Omega}\left[\left(M_{\alpha \beta, \beta}-Q_{\alpha}\right) W_{\alpha}^{*}+\left(Q_{\alpha, \alpha}+q\right) W_{3}^{*}\right] d \Omega=0, \\
\int_{\Omega}\left(N_{\alpha \beta, \beta}\right) U_{\alpha}^{*} d \Omega=0,
\end{array}
$$

where $W_{i}^{*}(i=\alpha, 3)$ and $U_{\alpha}^{*}$ are weight functions and $\Omega$ is the domain where integration takes place. Equation (13) is the integral representation associated with the governing equation for the results of bending and shear stress, while (14) is associated with the governing equation for the resultant membrane stresses.

2.2. Focus on Initial Stresses. In this section, we introduce the basic equation of elastoplastic problems in continuous media. Plasticity is a phenomenon independent of time and admits increases in load as a variation from one state to another. For example, the stress for the load step $(k)$ can be represented as

$$
\sigma_{i j}^{k}=\sigma_{i j}^{k-1}+\Delta \sigma_{i j} .
$$

In general terms, the nonlinearity of the material for flexural plates is considered by the incorporation of plastic deformations due to flexion and membrane [12]. Thus, strain ratios can be generically defined as

$$
\begin{aligned}
& \chi_{\alpha \beta}=\chi_{\alpha \beta}^{e}+\chi_{\alpha \beta}^{p} ; \\
& \varepsilon_{\alpha \beta}=\varepsilon_{\alpha \beta}^{e}+\varepsilon_{\alpha \beta}^{p} ; \\
& \gamma_{\alpha \beta}=\gamma_{\alpha \beta}^{e},
\end{aligned}
$$

where the superscripts " $e$ " and " $p$ " represent the elastic and plastic parts, respectively. 
On the other hand, the results of stresses can be established as

$$
\begin{gathered}
M_{\alpha \beta}^{e}=M_{\alpha \beta}+M_{\alpha \beta}^{p} ; \\
Q_{\alpha}=Q_{\alpha} ; \\
N_{\alpha \beta}^{e}=N_{\alpha \beta}+N_{\alpha \beta}^{p} .
\end{gathered}
$$

Equations (19), (20), and (21) represent the resulting stresses due to the momentum and membrane moment, respectively. The stress-displacement relations can be represented by the phenomenon of plasticity as

$$
\begin{aligned}
M_{\alpha \beta} & =D \frac{1-v}{2}\left(w_{\alpha, \beta}+w_{\beta, \alpha}+\frac{2 v}{1-\nu} w_{\gamma, \gamma} \delta_{\alpha \beta}\right)-M_{\alpha \beta}^{p} \\
Q_{\alpha} & =C\left(w_{\alpha}+w_{3, \alpha}\right) ; \\
N_{\alpha \beta} & =B \frac{1-v}{2}\left(u_{\alpha, \beta}+u_{\beta, \alpha}+\frac{2 \nu}{1-\nu} u_{\gamma, \gamma} \delta_{\alpha \beta}\right)-N_{\alpha \beta}^{p} .
\end{aligned}
$$

2.3. Integral Equations of Elastoplastic Strain. Here, we formulate the integral displacement equations for Reissner plates in materials with nonlinear behavior (i.e., plastic behavior). We begin by considering the equations of equilibrium (4)-(6) to have the following relations:

$$
\begin{array}{r}
\int_{\Omega}\left[\left(M_{\alpha \beta, \beta}-Q_{\alpha}\right) W_{\alpha}^{*}+\left(Q_{\alpha, \alpha}+q_{3}\right) W_{3}^{*}\right] d \Omega=0 \\
\int_{\Omega} N_{\alpha \beta, \beta} U_{\alpha}^{*} d \Omega=0
\end{array}
$$

where $W_{i}^{*}$ and $U_{\alpha}^{*}$ are the weight functions and $\Omega$ is the domain where the integration takes place. The above equations (25) and (26) together with Betti's reciprocal theorem are used to obtain the integral equations of displacement.

2.4. Integral Representation of Rotation and Deflection. The integral representing the ratio of rotations $\left(\omega_{\alpha}\right)$ and the deflection $\left(\omega_{3}\right)$ can be obtained by integrating $(25)$ by parts and applying Green's identity to obtain

$$
\begin{aligned}
& w_{i}\left(\mathbf{X}^{\prime}\right)+\int_{\Gamma} P_{i j}^{*}\left(\mathbf{X}^{\prime}, \mathbf{x}\right) w_{j}(\mathbf{x}) d \Gamma \\
& =\int_{\Gamma} W_{i j}^{*}\left(\mathbf{X}^{\prime}, \mathbf{x}\right) p_{j}(\mathbf{x}) d \Gamma \\
& \quad+\int_{\Omega} W_{i 3}^{*}\left(\mathbf{X}^{\prime}, \mathbf{X}\right) q_{3}(\mathbf{X}) d \Omega \\
& \quad+\int_{\Omega} \chi_{i \alpha \beta}^{*}\left(\mathbf{X}^{\prime}, \mathbf{X}\right) M_{\alpha \beta}^{P}(\mathbf{X}) d \Omega
\end{aligned}
$$

where $\mathbf{x} \in \Gamma$ and $\mathbf{X} \in \Omega$ are the field points on the boundary and the domain, respectively, and $W_{i j}^{*}\left(\mathbf{X}^{\prime}, \mathbf{x}\right), P_{i j}^{*}\left(\mathbf{X}^{\prime}, \mathbf{x}\right)$, and $x_{i \alpha \beta}^{*}\left(\mathbf{X}^{\prime}, \mathbf{x}\right)$ are the fundamental solutions of displacements, tractions, and strains, respectively.
2.5. Integral Representations of In-Plane Displacements. The integral representations related to the governing equations for the resulting membrane stresses can be obtained by integrating (26) by parts, and applying Green's identity gives

$$
\begin{aligned}
& u_{\theta}\left(\mathbf{X}^{\prime}\right)+\int_{\Gamma} T_{\theta \alpha}^{*}\left(\mathbf{X}^{\prime}, \mathbf{x}\right) u_{\alpha}(\mathbf{x}) d \Gamma \\
& =\int_{\Gamma} U_{\theta \alpha}^{*}\left(\mathbf{X}^{\prime}, \mathbf{x}\right) t_{\alpha}(\mathbf{x}) d \Gamma \\
& \quad+\int_{\Omega} \varepsilon_{\theta \alpha \beta}^{*}\left(\mathbf{X}^{\prime}, \mathbf{X}\right) N_{\alpha \beta}^{p}(\mathbf{X}) d \Omega,
\end{aligned}
$$

where $U_{\theta \alpha}^{*}\left(\mathbf{X}^{\prime}, \mathbf{x}\right), T_{\theta \alpha}^{*}\left(\mathbf{X}^{\prime}, \mathbf{x}\right)$, and $\varepsilon_{\theta \alpha \beta}^{*}\left(\mathbf{X}^{\prime}, \mathbf{x}\right)$ are the fundamental solutions of displacements, tractions, and strains, respectively.

2.6. Integral Boundary Equations of Displacement. In order to obtain solutions for points on the boundary, it is necessary to consider the limit $\mathbf{X}^{\prime} \longrightarrow x^{\prime} \in \Gamma$ in such a way that the boundary equation of the displacements can be written as

$$
\begin{gathered}
c_{i j} w_{i}\left(\mathbf{x}^{\prime}\right)+f_{\Gamma} P_{i j}^{*}\left(\mathbf{x}^{\prime}, \mathbf{x}\right) w_{j}(\mathbf{x}) d \Gamma \\
=\int_{\Gamma} W_{i j}^{*}\left(\mathbf{x}^{\prime}, \mathbf{x}\right) p_{j}(\mathbf{x}) d \Gamma \\
+\int_{\Omega} W_{i 3}^{*}\left(\mathbf{x}^{\prime}, \mathbf{X}\right) q_{3}(\mathbf{X}) d \Omega \\
+\int_{\Omega} \chi_{i \alpha \beta}^{*}\left(\mathbf{x}^{\prime}, \mathbf{x}\right) M_{\alpha \beta}^{P}(\mathbf{X}) d \Omega, \\
c_{\theta \alpha} u_{\theta}\left(\mathbf{x}^{\prime}\right)+f_{\Gamma} T_{\theta \alpha}^{*}\left(\mathbf{x}^{\prime}, \mathbf{x}\right) u_{\alpha}(\mathbf{x}) d \Gamma \\
=\int_{\Gamma} U_{\theta \alpha}^{*}\left(\mathbf{x}^{\prime}, \mathbf{x}\right) t_{\alpha}(\mathbf{x}) d \Gamma \\
+\int_{\Omega} \varepsilon_{\theta \alpha \beta}^{*}\left(\mathbf{x}^{\prime}, \mathbf{x}\right) N_{\alpha \beta}^{P}(\mathbf{X}) d \Omega
\end{gathered}
$$

where $f$ represents the principal value of Cauchy, $\mathbf{x}^{\prime}, \mathbf{x} \in \Gamma$ are the source and field points, respectively, on the boundary, and $c_{i j}\left(\mathbf{x}^{\prime}\right)$ and $c_{\theta \alpha}\left(\mathbf{x}^{\prime}\right)$ are the jump terms. The values of $c_{i j}\left(x^{\prime}\right)$ and $c_{\theta \alpha}\left(x^{\prime}\right)$ are equal to $(1 / 2) \delta_{i j}$ and $(1 / 2) \delta_{\theta \alpha}$, respectively, when $\mathbf{x}^{\prime}$ is located on a smooth boundary.

2.7. Integral Equations of Elastoplastic Stresses. The results of elastoplastic stresses on the points of the domain $\mathbf{X} \in \Omega$ can be evaluated by differential equations with respect to the source point $\mathbf{X}^{\prime}$. Finally, the integral equation for elastoplastic stresses can be written as

$$
\begin{aligned}
M_{\alpha \beta}\left(\mathbf{X}^{\prime}\right)= & \int_{\Gamma} W_{\alpha \beta k}^{*}\left(\mathbf{X}^{\prime}, \mathbf{x}\right) p_{k}(\mathbf{x}) d \Gamma \\
& -\int_{\Gamma} P_{\alpha \beta k}^{*}\left(\mathbf{X}^{\prime}, \mathbf{x}\right) w_{k}(\mathbf{x}) d \Gamma \\
& +\int_{\Omega} W_{\alpha \beta 3}^{*}\left(\mathbf{X}^{\prime}, \mathbf{X}\right) q_{3}(\mathbf{X}) d \Omega
\end{aligned}
$$




$$
\begin{aligned}
& +\int_{\Omega} \chi_{\alpha \beta \gamma \theta}^{*}\left(\mathbf{X}^{\prime}, \mathbf{X}\right) M_{\gamma \theta}^{p}(\mathbf{X}) d \Omega \\
& -\frac{\left[2(1+\nu) M_{\alpha \beta}^{p}+(1-3 v) M_{\theta \theta}^{p} \delta_{\alpha \beta}\right]}{8}
\end{aligned}
$$

For the result of stress due to moments,

$$
\begin{aligned}
Q_{\alpha}\left(\mathbf{X}^{\prime}\right)= & \int_{\Gamma} W_{3 \beta k}^{*}\left(\mathbf{X}^{\prime}, \mathbf{x}\right) p_{k}(\mathbf{x}) d \Gamma \\
& -\int_{\Gamma} P_{3 \beta k}^{*}\left(\mathbf{X}^{\prime}, \mathbf{x}\right) w_{k}(\mathbf{x}) d \Gamma \\
& +\int_{\Omega} W_{3 \beta 3}^{*}\left(\mathbf{X}^{\prime}, \mathbf{X}\right) q_{3}(\mathbf{X}) d \Omega \\
& +\int_{\Omega} \chi_{3 \beta \gamma \theta}^{*}\left(\mathbf{X}^{\prime}, \mathbf{X}\right) M_{\gamma \theta}^{P}(\mathbf{X}) d \Omega
\end{aligned}
$$

resulting from shear forces, and

$$
\begin{aligned}
N_{\alpha \beta}\left(\mathbf{X}^{\prime}\right)= & \int_{\Gamma} U_{\alpha \beta \gamma}^{*}\left(\mathbf{X}^{\prime}, \mathbf{x}\right) t_{\gamma}(\mathbf{x}) d \Gamma \\
& -\int_{\Gamma} T_{\alpha \beta \gamma}^{*}\left(\mathbf{X}^{\prime}, \mathbf{x}\right) u_{\gamma}(\mathbf{x}) d \Gamma \\
& +\int_{\Omega} \varepsilon_{\alpha \beta \gamma \theta}^{*}\left(\mathbf{X}^{\prime}, \mathbf{X}\right) N_{\gamma \theta}^{p}(\mathbf{X}) d \Omega \\
& -\frac{\left[2(1+\nu) N_{\alpha \beta}^{p}+(1-3 \nu) N_{\theta \theta}^{p} \delta_{\alpha \beta}\right]}{8}
\end{aligned}
$$

For the result of membrane stresses where the kernels $W_{i \beta k}^{*}, P_{i \beta k}^{*}$ are a linear combination of the first derivative of $W_{i j}^{*}$ and $P_{i j}^{*}$, the kernels $U_{\alpha \beta \gamma}^{*}, T_{\alpha \beta \gamma}^{*}$ are a linear combination of the first derivative of $U_{\alpha \beta}^{*}$ and $T_{\alpha \beta}^{*}$. The kernels $\chi_{i \beta \gamma \theta}^{*}, \varepsilon_{i \beta \gamma \theta}^{*}$ are a linear combination of the first derivative of $\chi_{i \alpha \beta}^{*}$ and $\varepsilon_{\alpha \beta \gamma}^{*}$.

We used BIE for in-plane stress/strain because it is considered that the plastic zone is not only because of bending but also because of in-plane load. On the other hand, the relation between the boundary integral equations for plate bending and plane strain is included in (31), (32), and (33) of the paper, where the last integral of each equations involves the nonlinear strain. Equation (31) represents stresses due to moments that cause plate bending, (32) denotes the stresses resulting from shear forces, and (33) characterizes the membrane stresses.

The above equations represent the integral boundary formulation for Reissner plates with large deformation and plastic behavior.

\section{Verification of the Method}

In order to verify the application and precision of the method presented in this paper, the problem solved by Karam and Telles [12] has been selected. This consists of a square plate (simply supported plate at its boundary) with dimensions such that the ratio $h / a=0.01$, where $h$ is the plate thickness

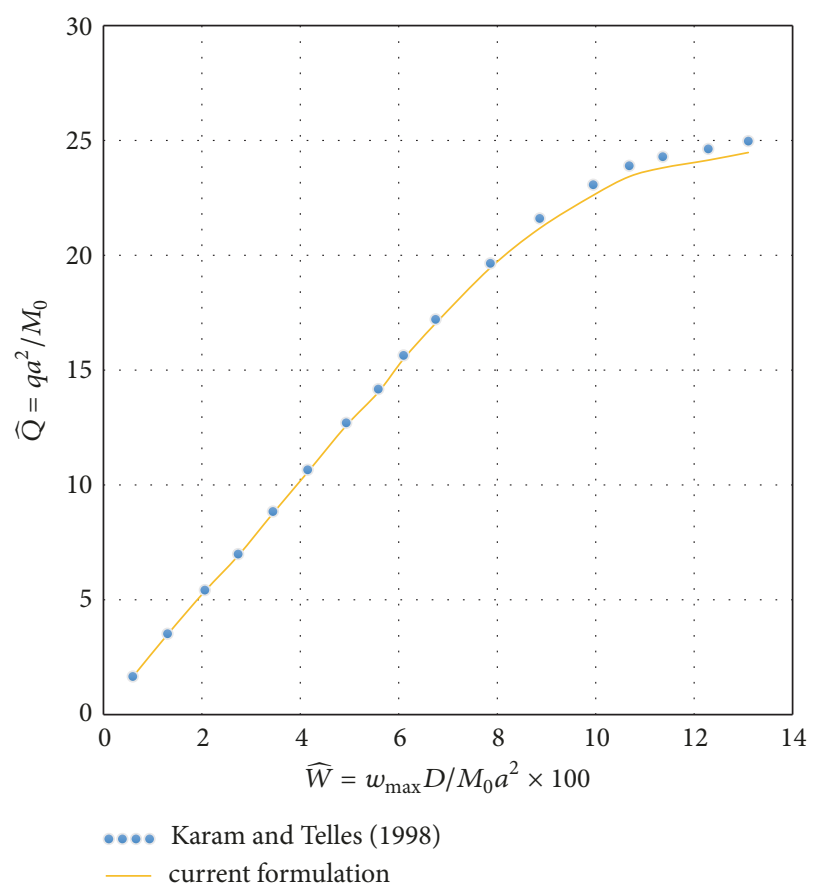

Figure 3: Deflection in the center of a square plate with uniform load. The symbols represent the results of Karam and Telles [12] and the continuous line represents those obtained by the present formulation.

and $a$ is the plate width; the plate is subjected to a uniform load in the vertical direction. The plate properties are $E=$ $10.92 \mathrm{GPa}, v=0.3$, and $\sigma_{Y}=1600 \mathrm{MPa}$. For these problems, the material is considered with elasto-perfectly plastic behavior (no hardening is considered). In Figure 3 we use the nondimensional parameters $\widehat{Q}=q a^{2} / M_{0}$ and $\widehat{W}=10^{2} w_{\max } D / M_{0} a^{2}$, where $M_{0}=\sigma_{Y} h^{2} / 4$ and $D=$ $E h^{2} /(1-v)$. The variable $q$ represents the uniform load applied to the plate. Figure 3 shows the results obtained by the present formulation and those obtained by Karam and Telles [12]. Good agreement between both formulations is observed. This figure shows the dimensionless deflection calculated at the center of the plate for several values of $\widehat{Q}$ load, which reaches a maximum value of 24.97. The results clearly illustrate a linear behavior and subsequently the plastic behavior of the plate.

\section{Probability of Failure in a Structural Element}

A structural element or system is designed to work in service conditions (elastic range) below the load for which it was designed (i.e., own weight, dead load, live load, earthquake, wind, and waves) for a certain interval of time. If an extraordinary load occurs before or after said time interval, there is a high probability that the structural element incurs in the inelastic range and the failure could occur. According to the above, it is important to consider such loads within the analysis in order to estimate the probability of failure of the structural element. One of the advantages of estimating the 
probability of failure is that it allows the redesign of the structural element so that it implicitly considers all possible magnitudes of loads, where a structural element would be safety. The disadvantage is that such element would be robust and costly.

The probability of failure of a structural element can be obtained by evaluating both the load solicitations (demand) and the ultimate capacity. The relationship between demand and capacity expressed in terms of a damage indicator (i.e., stress and deformation) is called damage index. The damage index has the advantage of normalizing the damage indicator with values between 0 and 1 and it permits quantifying the probability of no damage, the probability of damage, and the probability of failure. For the purpose of establishing a damage index, several authors have proposed damage indexes for structures subjected to earthquakes [31-36]. With the aim to estimate the probability of failure in Reissner's plates, a plastic behavior index that relates the relationship between the requested dimensionless displacement and the ultimate displacement is proposed as follows:

$$
I_{\mathrm{PB}}=\frac{\widehat{W}_{D}-\widehat{W}_{f}}{\widehat{W}_{F}-\widehat{W}_{f}},
$$

where $\widehat{W}_{D}$ is the requested dimensionless displacement; $\widehat{W}_{f}$ is the dimensionless displacement, where the plastic behavior of the structural element begins; $\widehat{W}_{F}$ is the ultimate capacity value of the structural element. According to the above, values of $I_{\mathrm{PB}}=0$ indicate that the structural element has not entered the plastic range and values of $I_{\mathrm{PB}}=1$ indicate the failure of the structural element.

Based on the plastic behavior index, $I_{\mathrm{PB}}$, it is possible to quantify empirically the probability of failure by a sample of Monte Carlo simulation as the sum of failures, $I_{\mathrm{PB}}=1=F$, between the number of cases, $N$; that is, $P_{F}=\sum_{i=1}^{N}(F / N)$. Therefore, the mean of the natural logarithm, $\mu_{\ln (F)}$, and the standard deviation of the natural logarithm, $\sigma_{\ln (F)}$, are obtained as follows:

$$
\begin{aligned}
& \mu_{\ln (F)}=\exp \left(\frac{\sum_{i=1}^{N} \ln (F)}{N}\right) \\
& \sigma_{\ln (F)}=\left(\frac{\sum_{i=1}^{N}\left(\ln (F)-\ln \left(\mu_{\ln (F)}\right)\right)^{2}}{N-1}\right)^{1 / 2}
\end{aligned}
$$

If failures of the structural element follow a lognormal distribution [37], they can be fitted theoretically as follows:

$$
\begin{aligned}
P_{F} & =P(F \geq 1)=\int f_{F}(F) d F \\
& =\int \frac{1}{\sigma_{\ln (F)} F} \varphi\left(\frac{\ln (F)-\ln \left(\mu_{\ln (F)}\right)}{\sigma_{\ln (F)}}\right) d F \\
& =\Phi\left(\frac{\ln F-\mu_{\ln (F)}}{\sigma_{\ln (F)}}\right)
\end{aligned}
$$

where $\varphi$ is the normal distribution function; $\Phi$ is the standard normal probability distribution function.

\section{Methodology}

The probability of failure of the plate due to loads is studied by means of a Monte Carlo simulation. The methodology is described in Figure 4.

\section{Application Example}

In this section, the failure probability of a plate is evaluated considering the uncertainties in its mechanical (yielding, ultimate stress, and modulus of elasticity) and geometrical (thickness, length, and width) properties. The nominal properties of the plate are the following: $0.1 \mathrm{~m}$ long by $0.1 \mathrm{~m}$ wide, with the thickness being $0.05 \mathrm{~m}$. The yield stress is $248.108 \mathrm{MPa}$, the ultimate stress is $400.111 \mathrm{MPa}$, and the modulus of elasticity is $200055.66 \mathrm{MPa}$. Table 1 shows the statistical parameters reported by Mansour et al. [38] and Galambos and Ravindra [39].

The plastic behavior index and the probability of failure are obtained considering the procedure described in the previous section. 55 cases were simulated considering the geometric and mechanical uncertainties. It was verified that a larger number of samples did not change the statistical results. Figure 5 shows the plastic behavior index (see (34)) considering the simulated cases. It is noted that the variability of the curves of the plastic behavior index is due to the fact of considering the uncertainties in mechanical and geometric properties. According to the above, different $\widehat{W}$ values can be observed, where $I_{\mathrm{PB}}=1$, that is, different failure scenarios of the structural element. For $I_{\mathrm{PB}}=1, \widehat{W}$ values are between 1.26 and 2.38, which correspond to 0.023 and $0.039 \mathrm{~m}$, respectively. This range is similar to the displacement failure values reported by Rudrapatna et al. [40], where the authors studied a plate with similar geometric and mechanical characteristics using the finite element method and validated the results by experimentally testing the plate up to failure.

Figure 6 shows the probability of empirical and theoretical failure. It is noticed that the points of the empirical failure probability plot follow a lognormal distribution, so the assumption made in (37) is verified. Figure 6 displays that the probability of failure for $0<\widehat{W}<0.4$ is null. However, for values of $0.5<\widehat{W}<2.5$, failure probability increases as $\widehat{W}$ increases: $P_{F}=4.85 E-12$ and $P_{F}=9.99 E-01$, respectively. This range of failure probability can occur when the uncertainty in mechanical and geometric properties is considered. The failure event is imminent for $\widehat{W} \geq 2.5$, since empirical and theoretical probabilities of failure are the same: $P_{F}=1$.

\section{Conclusions}

In the present work, the application of the boundary element method is used to solve the nonlinear problem of plasticity in Reissner's plates. We use the classical theory of plasticity and a formulation of initial stresses, which allow the formulation of integral boundary equations due to plasticity. For the calculation of the plastic zone, the von Mises criterion is used. To verify the validity and accuracy of our formulation, 


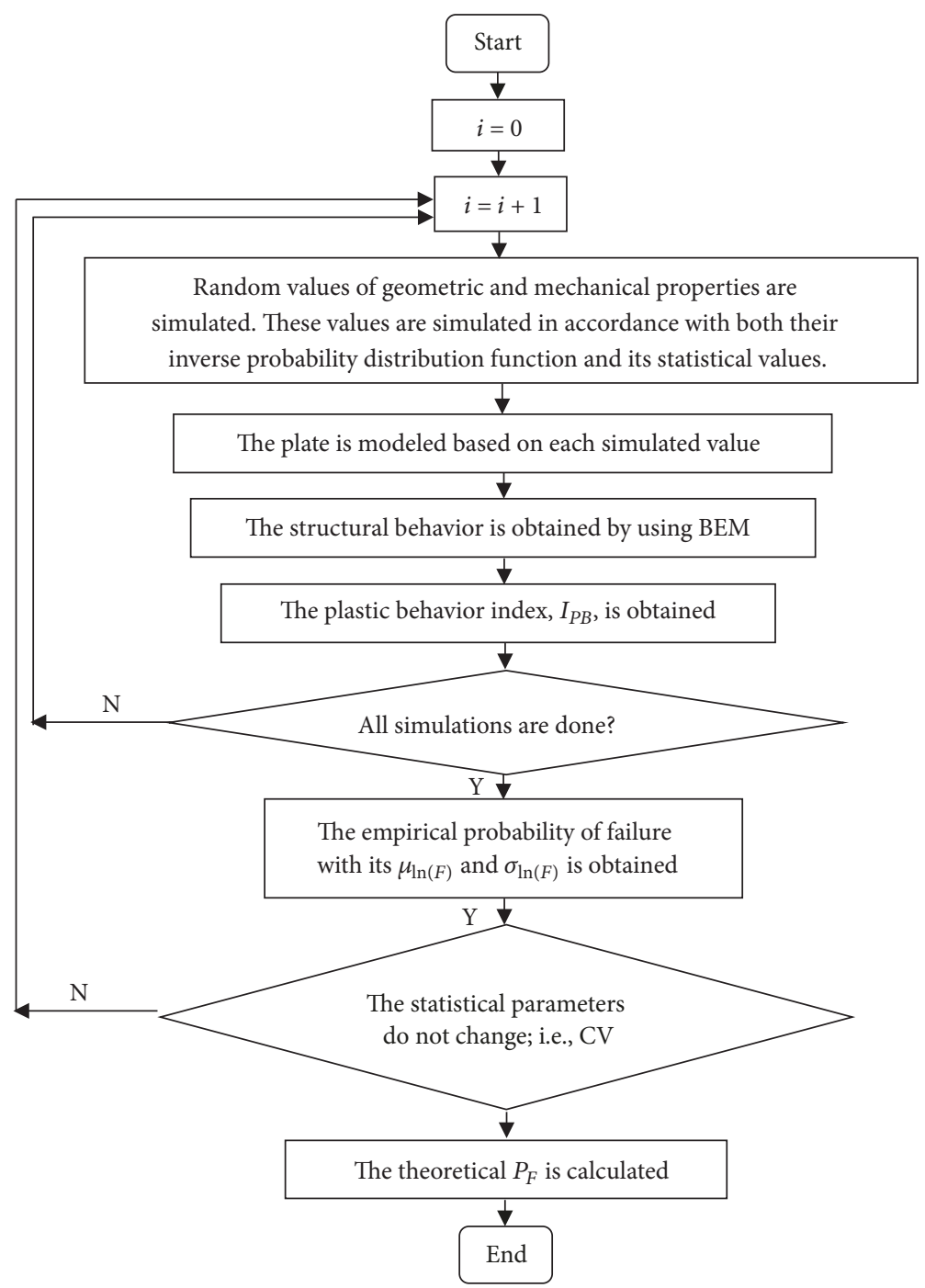

FIGURE 4: Flowchart for obtaining the probability of failure.

TABLE 1: Statistical parameters used in the present study.

\begin{tabular}{lccc}
\hline Variable & Mean value & Coefficient of variation $(\mathrm{CV})$ & Type of distribution \\
\hline$h$ & 1.05 & 0.044 & Lognormal \\
$a$ & 0.988 & 0.046 & Lognormal \\
$E$ & 0.987 & 0.076 & Lognormal \\
$F_{y}$ & 1.3 & 0.124 & Normal \\
$F_{u}$ & 1.05 & 0.075 & Normal \\
\hline
\end{tabular}

the results were compared with those obtained by Karam and Telles [12]. The plastic behavior index proposed in this work to quantify the probability of failure has been validated against published data.

\section{Data Availability}

The data used to support the findings of this study are available from the corresponding author upon request.

\section{Conflicts of Interest}

The authors declare that there are no conflicts of interest regarding the publication of this paper.

\section{Acknowledgments}

The authors are grateful for the support granted by CONACYT under the Project CB-2014/240413 and they acknowledge Instituto Politécnico Nacional for the support. 


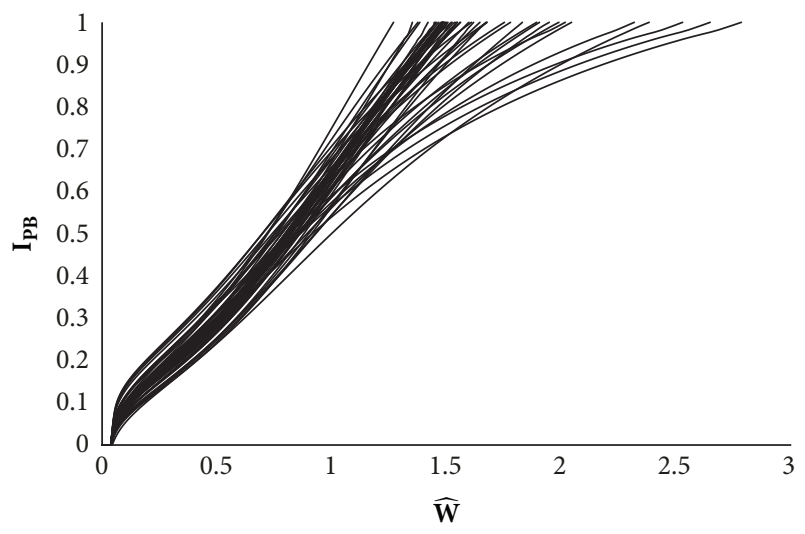

Figure 5: Plastic behavior index for the 20 considered cases.

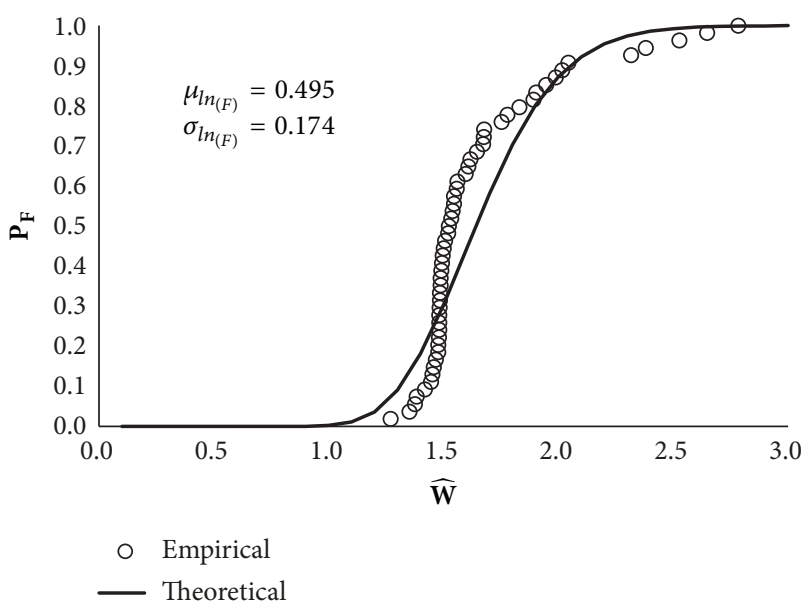

FIgURE 6: Probability of empirical and theoretical failure.

\section{References}

[1] E. Reissner, "On a Variational Theorem in Elasticity," Journal of Mathematics and Physics, vol. 29, pp. 90-95, 1950.

[2] R. D. Mindlin, "Influence of Rotatory Inertia and Shear on Flexural Motions of Isotropic, Elastic Plates," Journal of Applied Mechanics, vol. 18, pp. 31-38, 1951.

[3] S. Timoshenko and J. N. Goodier, Theory of Elasticity, McGrawHill International Editions, Springer, Berlin, Germany, 3rd edition, 1970

[4] A. Mendelson, Plasticity: Theory and Application, Macmillan, New york, NY, USA, 1968.

[5] R. Hill, The Mathematical Theory of Plasticity, Clarendon Press, Oxford, UK, 1950.

[6] W. Prager, An Introduction to Plasticity, Addison-Wesley, Amsterdam, Netherlands, 1959.

[7] H.-S. Shen, J. Yang, and L. Zhang, "Dynamic response of Reissner-Mindlin plates under thermomechanical loading and resting on elastic foundations," Journal of Sound and Vibration, vol. 232, no. 2, pp. 309-329, 2000.

[8] P. H. Wen, "The fundamental solution of Mindlin plates resting on an elastic foundation in the Laplace domain and its applications," International Journal of Solids and Structures, vol. 45, no. 3-4, pp. 1032-1050, 2008.
[9] P. H. Wen and M. H. Aliabadi, "Boundary element formulations for Mindlin plate on an elastic foundation with dynamic load," Engineering Analysis with Boundary Elements, vol. 33, no. 10, pp. 1161-1170, 2009.

[10] V. Jankovski and V. Skaržauskas, "The physically nonlinear analysis of circular plate on deformable foundation," The Baltic Journal of Road and Bridge Engineering, vol. 6, no. 1, pp. 59-66, 2011.

[11] A. Nosier and F. Fallah, "Reformulation of Mindlin-Reissner governing equations of functionally graded circular plates," Acta Mechanica, vol. 198, no. 3-4, pp. 209-233, 2008.

[12] V. J. Karam and J. C. F. Telles, "Nonlinear material analysis of Reissners plates, Plate Bending Analysis with Boundary Element, Computational Mechanics Publications," Nonlinear material analysis of Reissners plates, Plate Bending Analysis with Boundary Element, Computational Mechanics Publications, 1998.

[13] X.-Y. Lei, M.-K. Huang, and X. Wang, "Geometrically nonlinear analysis of a Reissner type plate by the boundary element method," Computers \& Structures, vol. 37, no. 6, pp. 911-916, 1990.

[14] M. A. Benatta, A. Kaci, A. Tounsi, M. S. Houari, K. Bakhti, and E. A. Adda Bedia, "Nonlinear bending analysis of functionally graded plates under pressure loads using a four variable refined plate theory," International Journal of Computational Methods, vol. 11, no. 4, Article ID 1350062, 1350062, 16 pages, 2014.

[15] F. G. Yuan and S. Yang, "Asymptotic crack-tip fields in an anisotropic plate subjected to bending, twisting moments and transverse shear loads," Composites Science and Technology, vol. 60, no. 12-13, pp. 2489-2502, 2000.

[16] J. G. Simmonds, "Pointwise errors in the classical and in Reissner's linear theory of plates, especially for concentrated loads," Journal of Elasticity: The Physical and Mathematical Science of Solids, vol. 23, no. 2-3, pp. 219-232, 1990.

[17] W. Yu and D. H. Hodges, "A simple thermopiezoelastic model for smart composite plates with accurate stress recovery," Smart Materials and Structures, vol. 13, no. 4, pp. 926-938, 2004.

[18] P. Manríquez, E. Contreras-Reyes, and A. Osses, "Lithospheric 3-D flexure modelling of the oceanic plate seaward of the trench using variable elastic thickness," Geophysical Journal International, vol. 196, no. 2, pp. 681-693, 2013.

[19] M. Ettouney, H. Benaroya, and J. Wright, "Boundary element methods in probabilistic structural analysis (PBEM)," Applied Mathematical Modelling, vol. 13, no. 7, pp. 432-441, 1989.

[20] I. Kaljević and S. Saigal, "Stochastic boundary elements in elastostatics," Computer Methods Applied Mechanics and Engineering, vol. 109, no. 3-4, pp. 259-280, 1993.

[21] M. Kaminski, "Stochastic second-order BEM perturbation formulation," Engineering Analysis with Boundary Elements, vol. 23, no. 2, pp. 123-129, 1999.

[22] M. Kamiński, "Application of the generalized perturbationbased stochastic boundary element method to the elastostatics," Engineering Analysis with Boundary Elements, vol. 31, no. 6, pp. 514-527, 2007.

[23] X. Huang and M. H. Aliabadi, "Probabilistic fracture mechanics by the boundary element method," International Journal of Fracture, vol. 171, no. 1, pp. 51-64, 2011.

[24] G. Chen, S. Rahman, and Y. H. Park, "Shape sensitivity and reliability analyses of linear-elastic cracked structures," International Journal of Fracture, vol. 112, no. 3, pp. 223-246, 2001. 
[25] C. Su and C. Zheng, "Probabilistic fracture mechanics analysis of linear-elastic cracked structures by spline fictitious boundary element method," Engineering Analysis with Boundary Elements, vol. 36, no. 12, pp. 1828-1837, 2012.

[26] C. Su, S. Zhao, and H. Ma, "Reliability analysis of plane elasticity problems by stochastic spline fictitious boundary element method," Engineering Analysis with Boundary Elements, vol. 36, no. 2, pp. 118-124, 2012.

[27] C. Su and J. Xu, "Reliability analysis of Reissner plate bending problems by stochastic spline fictitious boundary element method," Engineering Analysis with Boundary Elements, vol. 51, pp. 37-43, 2015.

[28] D. W. Kim and B. M. Kwak, "Reliability-based shape optimization of two-dimensional elastic problems using BEM," Computers \& Structures, vol. 60, no. 5, pp. 743-750, 1996.

[29] Supriyono and M. H. Aliabadi, "Boundary element method for shear deformable plates with combined geometric and material nonlinearities," Engineering Analysis with Boundary Elements, vol. 30, no. 1, pp. 31-42, 2006.

[30] T. Dirgantara and M. H. Aliabadi, "Elastoplastic boundary element method for shear deformable shells," Engineering Structures, vol. 45, pp. 62-67, 2012.

[31] H. Banon and D. Veneziano, "Seismic safety of reinforced concrete members and structures," Earthquake Engineering \& Structural Dynamics, vol. 10, no. 2, pp. 179-193, 1982.

[32] M. S. L. Roufaiel and C. Meyer, "Analytical modeling of hysteretic behavior of R/C frames," Journal of Structural Engineering (United States), vol. 113, no. 3, pp. 429-444, 1987.

[33] E. Cosenza, G. Manfredi, and R. Ramasco, "The use of damage functionals in earthquake engineering: A comparison between different methods," Earthquake Engineering \& Structural Dynamics, vol. 22, no. 10, pp. 855-868, 1993.

[34] J. M. Bracci, A. M. Reinhorn, and J. B. Mander, "Deterministic model for seismic damage evaluation of reinforced concrete structures," Tech. Rep., State University of New York at Buffalo, 1989.

[35] Y.-J. Park and A. H.-S. Ang, "Mechanistic seismic damage model for reinforced concrete," Journal of Structural Engineering, vol. 111, no. 4, pp. 722-739, 1985.

[36] G. S. Kamaris, G. D. Hatzigeorgiou, and D. E. Beskos, "A new damage index for plane steel frames exhibiting strength and stiffness degradation under seismic motion," Engineering Structures, vol. 46, pp. 727-736, 2013.

[37] E. Rosenblueth and L. Esteva, "Reliability basis for some Mexican codes," in in Probabilistic Design of Reinforced Concrete Buildings, ACI Publication SP-31, American Concrete Institute, p. 41, MI, Farmington Hills, 1972.

[38] A. E. Mansour, H. Y. Jan, C. I. Zigelman, Y. N. Chen, and S. J. Harding, "Implementation of reliability methods to marine structures," Transactions Society of Naval Architects and Marine Engineers, vol. 92, pp. 353-382, 1984.

[39] T. V. Galambos and T. V. Ranvindra, Properties of steel for use in LRFD Journal of the Structural Division104, pp. 1459-1468, 1978.

[40] N. S. Rudrapatna, R. Vaziri, and M. D. Olson, "Deformation and failure of blast-loaded square plates," International Journal of Impact Engineering, vol. 22, no. 4, pp. 449-467, 1999. 


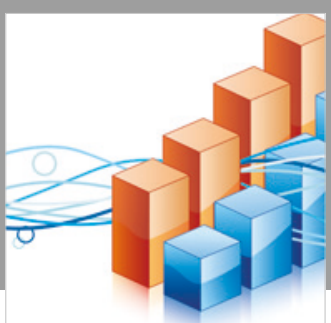

Advances in

Operations Research

\section{-n-m}
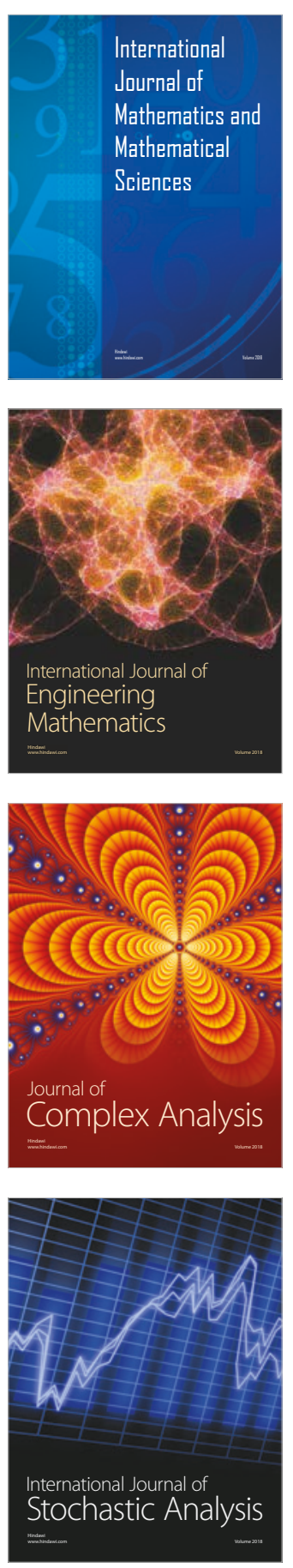
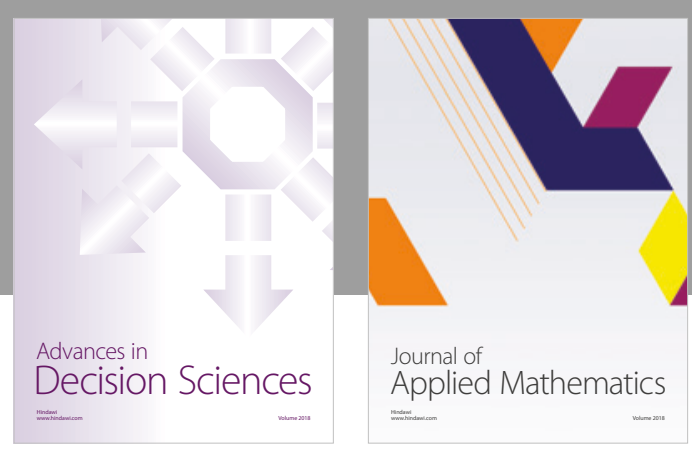

Journal of

Applied Mathematics
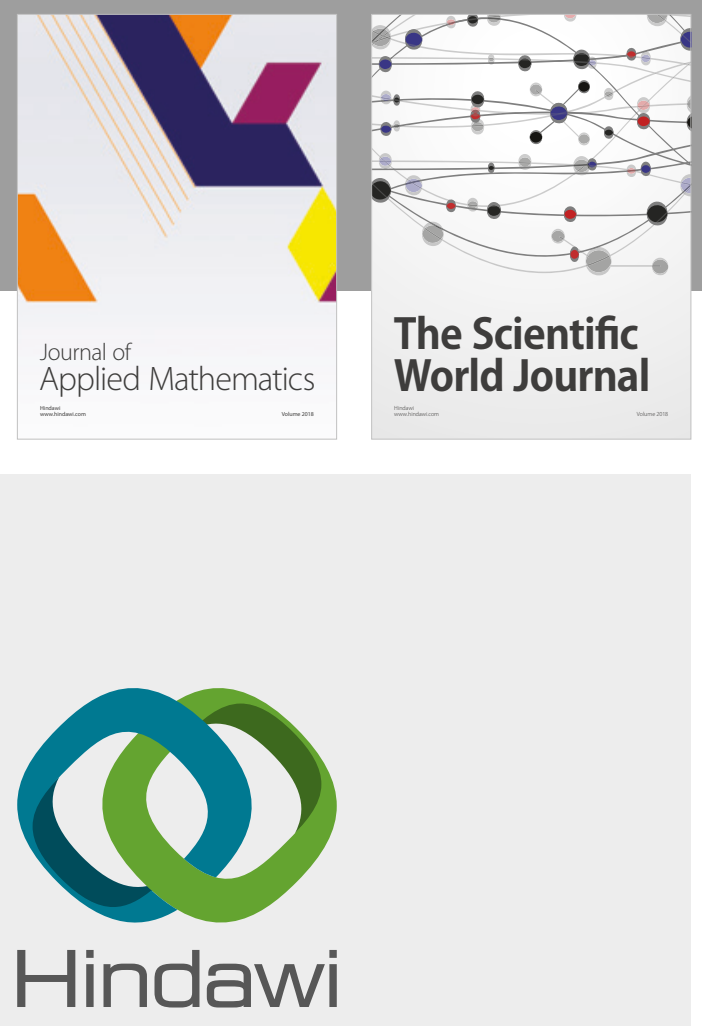

Submit your manuscripts at

www.hindawi.com

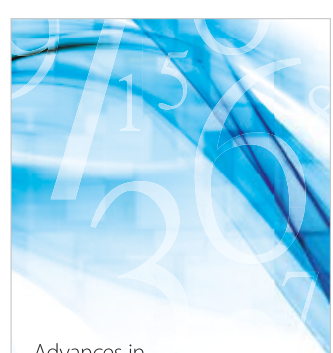

Advances in
Numerical Analysis
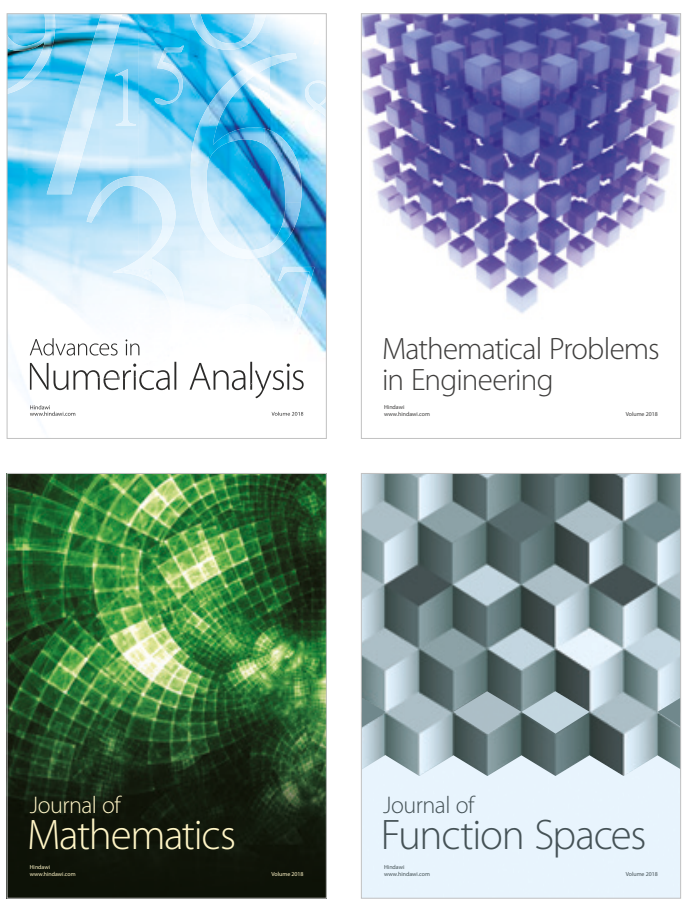

Mathematical Problems in Engineering

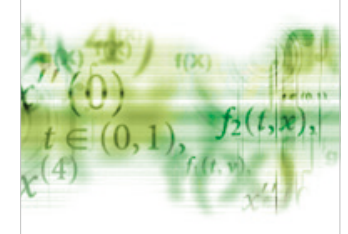

International Journal of

Differential Equations

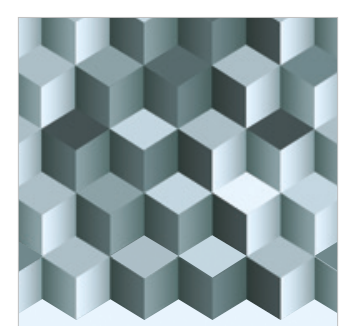

Journal of

Function Spaces

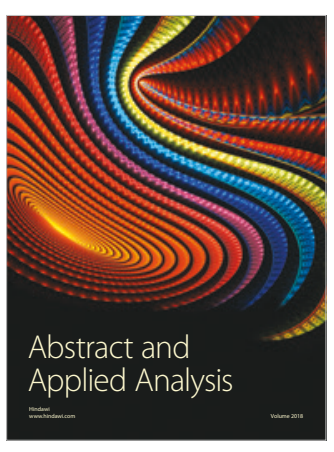

The Scientific

World Journal

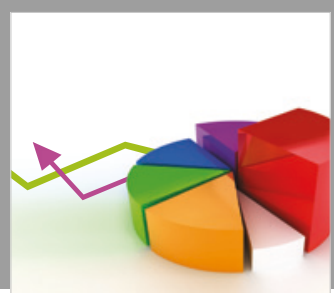

Journal of

Probability and Statistics
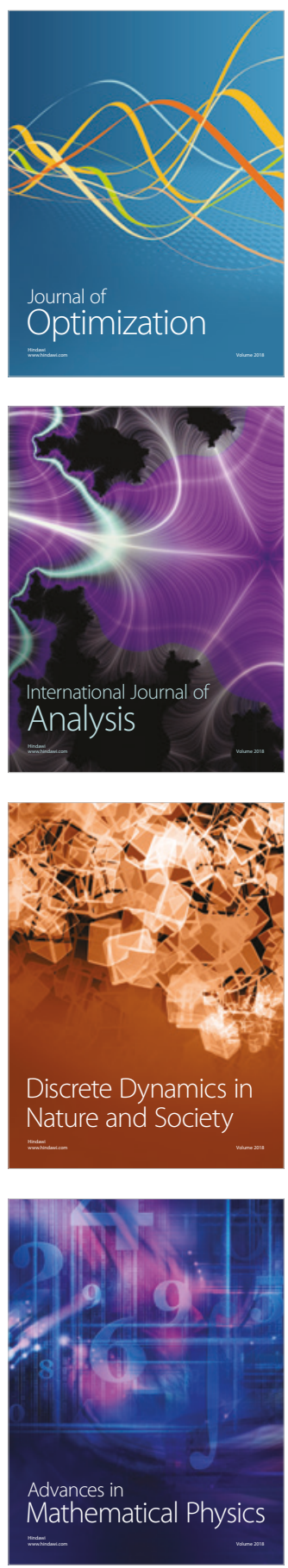